\title{
COUNTING BALANCED SIGNED GRAPHS USING MARKED GRAPHS
}

\author{
by FRANK HARARY and JERALD A. KABELL
}

(Received 30th November 1979)

Dedicated to Lowell W. Beineke

\section{Introduction}

A graph in which each line is designated as either positive or negative is called a signed graph $S$. The sign of a cycle in $S$ is a product of the signs of its lines. A signed graph in which every cycle is positive is called balanced. This concept was introduced by Harary in (3) and the following characterisation of balanced signed graphs was given.

Structure Theorem for Balance. A signed graph $S$ with at least one negative line is balanced if and only if the point set $V(S)$ can be partitioned into two parts in such a way that all the negative lines lie between the parts while the positive lines lie within the parts.

The psychological implications of this theorem were studied by Cartwright and Harary (2).

The problem of enumerating signed graphs was solved by Harary (4), but that of counting balanced signed graphs $S$ proved more resistant. Let the two parts of $S$ given by the Structure Theorem for Balance have cardinalities $m$ and $n$. Harary and Palmer (5) found a formula for the number $b_{m, n}$ of balanced signed graphs with $m, n$ as parameters. This formula is not satisfactory for calculating $b_{p}$, the number with $p$ points, as $b_{p}=\sum b_{m, n}$ over all $m, n$ with $m \leqq n$ and $m+n=p$. Our purpose is to determine directly a formula for $b_{\mathrm{p}}$ which is easily computable.

Recently, interest has focused on marked graphs, in which the points, rather than the lines, are designated positive and negative. The structure of these has been studied by Beineke and Harary (1), and the enumeration problem (along with that for several related structures) has been completely and elegantly solved by Harary, Palmer, Robinson and Schwenk (7). This work, together with a natural correspondence between marked graphs and balanced signed graphs, gives a counting formula for the latter.

\section{The correspondence between marked graphs and balanced signed graphs}

Given a marked graph $M$, it is natural to associate with it a signed graph, by taking the sign of each line to be the product of the signs of its two points. We explore this relationship more fully in the following result. The authors wish to thank Lászlo Babai for providing the key idea in the converse part of the theorem. 
We say that a marked graph $M$ and a signed graph $S$ both having the same unmarked unsigned underlying graph $G$ correspond if the sign of each line in $S$ is the product of the signs of its two points in $M$.

Correspondence Theorem. Each connected marked graph corresponds to precisely one balanced signed graph. Each connected balanced signed graph corresponds to two (possibly isomorphic) marked graphs.

Proof. Consider any connected marked graph $M$. To each line of $M$ assign the product of the signs of its points. The product of the line signs of any cycle is then the square of the product of the point signs, hence it must be positive. Thus the signed graph obtained by now erasing the point signs is balanced. It is clear of course that the dual $M^{*}$ (obtained by changing all the signs) of $M$ will yield the same signed graph.

Conversely, let $S$ be a connected balanced signed graph and $T$ a spanning tree of $S$. Root $T$ and assign a sign to the root arbitrarily. Now each point adjacent to the root is assigned the product of the sign of the root and the sign of the line joining it to the root. Similarly any point already signed can be used to generate appropriate signs for all of its (previously unsigned) neighbours. Continuing in this way, we can assign signs to all points of $S$ consistent with the line signs of $T$. Now consider any line not in $T$, say $u v$. There is a unique $u-v$ path in $T$, and since $S$ is balanced, the sign of $u v$ must be the product of all the line signs in the $u v$ path. By the procedure we have used to assign point signs, however, the product of the line signs of the $u-v$ path must be just the product of the sign of $u$ and the sign of $v$, hence the assignment of point signs is consistent with the given line signs of $S$. Again, it is clear that the opposite choice of sign for the root will yield the dual marking.

\section{Counting balanced signed graphs}

Explicitly, a marked graph $M$ is self-dual if it is isomorphic to its dual $M^{*}$. From the Correspondence Theorem it is now clear that there is a one-to-one correspondence between dual pairs of connected marked graphs (regarding a self-dual marked graph as both members of a dual pair) and connected balanced signed graphs. As both marked graphs and self-dual marked graphs have been enumerated by Harary, Palmer, Robinson and Schwenk (7), we can now exploit this correspondence to obtain counting formulas for balanced signed graphs and connected balanced signed graphs. The following generating functions will be useful in this endeavor (where $m_{p}$ is of the number of isomorphism classes of marked graphs of order $p$, and so forth):

$$
\begin{aligned}
& m(x)=\sum_{p=1}^{\infty} m_{p} x^{p} \quad \text { marked graphs, } \\
& m^{*}(x)=\sum_{p=1}^{\infty} m_{p}^{*} x^{p} \quad \text { self-dual marked graphs, } \\
& n(x)=\sum_{p=1}^{\infty} n_{p} x^{p} \quad \text { connected marked graphs, } \\
& n^{*}(x)=\sum_{p=1}^{\infty} n_{p}^{*} x^{p} \quad \text { connected self-dual marked graphs, }
\end{aligned}
$$




$$
\begin{array}{ll}
t(x)=\sum_{p=1}^{\infty} t_{p} x^{p} & \text { dual pairs of marked graphs, } \\
d(x)=\sum_{p=1}^{\infty} d_{p} x^{p} & \text { connected balanced signed graphs, } \\
b(x)=\sum_{p=1}^{\infty} b_{p} x^{p} & \text { balanced signed graphs. }
\end{array}
$$

Proposition 1. The generating functions $m(x)$ and $n(x)$ are related by

$$
1+m(x)=\exp \sum_{k=1}^{\infty} \frac{n\left(x^{k}\right)}{k} .
$$

Proof. Following the basic argument from Harary and Palmer (6, p. 90), in which $Z\left(S_{\infty}\right)$ stands for the sum of the cycle indexes of all the symmetric groups $S_{n}$, we first note that any marked graph can be regarded as a union of connected marked graphs. Thus the number of marked graphs with $k$ components is given by $Z\left(S_{k}, n(x)\right)$, so $1+m(x)=Z\left(S_{\infty}, n(x)\right)$ from which the desired result follows immediately by $(6, \mathrm{p}$. 52).

The actual calculation of the coefficients $n_{p}$ is facilitated by the introduction of an auxiliary series, defined by

$$
\sum_{p=1}^{\infty} a_{p} x^{p}=\log (1+m(x)) .
$$

From this we readily calculate coefficients $a_{\mathrm{p}}$ by

$$
a_{p}=m_{p}-\frac{1}{p} \sum_{k=1}^{p-1} k a_{k} m_{p-k} .
$$

In view of Proposition 1, we also have

$$
\sum_{p=1}^{\infty} a_{p} x^{p}=\sum_{p=1}^{\infty} \frac{n\left(x^{k}\right)}{k}
$$

from which

$$
a_{p}=\frac{1}{p} \sum_{r \mid p} m_{r}
$$

or

$$
n_{p}=\sum_{r / p} \frac{\mu(r)}{r} a_{p} / r
$$

where $\mu$ is the Möbius function. Combined with (1) this enables us to readily compute the numbers of connected marked graphs.

The situation for self-dual marked graphs is slightly more complicated, as reflected in the following result. 
Proposition 2. The generating functions $m^{*}(x), n^{*}(x)$ and $t(x)$ are related by

$$
1+m^{*}(x)=\exp \sum_{k=1}^{\infty} \frac{t\left(x^{k}\right)+n^{*}\left(x^{k}\right)}{k}
$$

Proof. A self-dual marked graph can be regarded as a union of two different marked graphs, one consisting of those components which are individually self-dual, and the other containing the remaining components which occur in dual pairs. The same argument used in Proposition 1 shows that marked graphs, each component of which is self-dual, are counted by $Z\left(S_{\infty}, n^{*}(x)\right)$, while those self-dual marked graphs with no self-dual components are given by $Z\left(S_{\infty}, t(x)\right)$. Combining these yields

$$
1+m^{*}(x)=\left(\exp \sum_{k=1}^{\infty} \frac{t\left(x^{k}\right)}{k}\right)\left(\exp \sum_{k=1}^{\infty} \frac{n^{*}\left(x^{k}\right)}{k}\right)
$$

from which the proposition follows easily.

As in the case of marked graphs, we introduce an auxiliary series to aid in the computation of the coefficients $n_{p}^{*}$ by defining

$$
\sum_{p=1}^{\infty} a_{p}^{*} x^{p}=\log \left(1+m^{*}(x)\right)
$$

As before we find

$$
a_{\mathrm{p}}^{*}=m_{\mathrm{p}}^{*}-\frac{1}{p} \sum_{k=1}^{p-1} k a_{\mathrm{k}}^{*} m_{\mathrm{p}-\mathrm{k}}^{*}
$$

and likewise,

$$
n_{p}^{*}+t_{p}=\sum_{r / p} \frac{\mu(r)}{r} a_{p / r}^{*} .
$$

What remains now is to determine $t_{p}$, but as

$$
t(x)=\frac{1}{2}\left(n\left(x^{2}\right)-n^{*}\left(x^{2}\right)\right)
$$

we obtain

$$
t_{\mathrm{p}}= \begin{cases}\frac{1}{2}\left(n_{\mathrm{p} / 2}-n_{\mathrm{p} / 2}^{*}\right) & \text { for } p \text { even } \\ 0 & \text { for } p \text { odd }\end{cases}
$$

At this point we can make the transition from marked graphs to balanced signed graphs.

Proposition 3. The number of connected balanced signed graphs is given by

$$
d(x)=\frac{1}{2}\left(n(x)+n^{*}(x)\right)
$$

Proof. In view of the Correspondence Theorem, we need only count the dual pairs of connected marked graphs, given by $\left(n(x)-n^{*}(x)\right) / 2$, and add the generating function $n^{*}(x)$ for self-dual connected marked graphs. 
The actual computation of the coefficients $d_{\mathrm{p}}$ is of course, quite straightforward, given by

$$
d_{\mathrm{p}}=\frac{1}{2}\left(n_{\mathrm{p}}+n_{\mathrm{p}}^{*}\right) \text {. }
$$

The final step, to balanced signed graphs from the connected species, is merely the reverse of Proposition 1, and the proof is precisely parallel.

Proposition 4. The generating functions $d(x)$ and $b(x)$ are related by

$$
\exp \sum_{k=1}^{\infty} \frac{d\left(x^{k}\right)}{k}=1+b(x)
$$

The computation is also the reverse of the step from marked graphs to connected marked graphs. The coefficients of the auxiliary series are defined by

$$
e_{\mathrm{p}}=\frac{1}{p} \sum_{r \mid \mathrm{p}} r d_{r}
$$

so that $1+b(x)=\exp \sum_{p=1}^{\infty} e_{p} x^{p}$, and the coefficients $b_{p}$ are given by

$$
b_{p}=e_{p}+\frac{1}{p} \sum_{k=1}^{p-1} k e_{k} b_{p-k} .
$$

Using the previously determined values of $m_{\mathrm{p}}$ and $m_{\mathrm{p}}^{*}$ from (7), and formulas (1)-(8) above, we can complete the following table which gives the numbers through six points.

Table 1. The numbers of balanced signed graphs, with other numbers needed to calculate these.

\begin{tabular}{rrrrrrrr}
\hline$p$ & $m_{\mathrm{p}}$ & $m_{\mathrm{p}}^{*}$ & \multicolumn{1}{c}{$n_{\mathrm{p}}$} & $n_{\mathrm{p}}^{*}$ & $t_{\mathrm{p}}$ & \multicolumn{1}{c}{$d_{\mathrm{p}}$} & \multicolumn{1}{c}{$b_{\mathrm{p}}$} \\
\hline 1 & 2 & 0 & 2 & 0 & 0 & 1 & 1 \\
2 & 6 & 2 & 3 & 1 & 1 & 2 & 3 \\
3 & 20 & 0 & 10 & 0 & 0 & 5 & 8 \\
4 & 90 & 10 & 50 & 6 & 1 & 28 & 39 \\
5 & 544 & 0 & 354 & 0 & 0 & 177 & 226 \\
6 & 5096 & 104 & 3883 & 81 & 5 & 1982 & 2283 \\
\hline
\end{tabular}

(The authors wish to thank Allen Schwenk for a helpful comment on an earlier version of this work).

\section{REFERENCES}

(1) L. W. Beineke and F. Harary, Consistent graphs with signed points, Rivista di matematica per le scienze economiche e sociale 1 (1978), 81-88.

(2) D. Cartwright and F. Harary, Structural balance: A generalization of Heider's theory, Psychol. Review 63 (1956), 277-293.

(3) F. Harary, On the notion of balance of a signed graph, Michigan Math. J. 2 (1953), 143-146. 
(4) F. Harary, The number of linear, directed, rooted, and connected graphs, Trans. Amer. Math. Soc. 78 (1955), 455-463.

(5) F. Harary and E. M. Palmer, On the number of balanced signed graphs, Bull. Math. Biophysics 29 (1967), 759-765.

(6) F. Harary and E. M. Palmer, Graphical Enumeration (Academic Press, New York, 1973).

(7) F. Harary, E. M. Palmer, R. W. Robinson and A. J. Schwenk, Enumeration of graphs with signed points and lines, J. Graph Theory 1 (1977), 295-308.

Department of Mathematics

UNIVERSITY OF MICHIGAN

ANN ARBOR, MI 48109 